\title{
TRIBUTOS, PIOJOS Y DIOSES: IMPLICANCIAS CULTURALES Y PREVALENCIA DE LA PEDICULOSIS EN INDIVIDUOS DEL SITIO INCAICO DE CAMARONES 9, NORTE DE CHILE
}

\author{
TAXES, HEAD LICE AND GODS: CULTURAL IMPLICATIONS AND \\ PREVALENCE OF PEDICULOSIS IN INDIVIDUALS FROM THE CAMARONES 9 \\ INCA SITE, NORTHERN CHILE
}

\author{
Bernardo Arriaza ${ }^{1}$, Natalia Aravena ${ }^{1}$,Hipólito Núñez $z^{2 \dagger}$ y Vivien G. Standen ${ }^{3}$
}

\begin{abstract}
En este trabajo se estudia la prevalencia de pediculosis en el sitio incaico Camarones 9 del extremo norte de Chile, con el propósito de contrastar lo descrito en las crónicas y lo observado en el registro bioarqueológico. La pediculosis es una ectoparasitosis frecuente, pero las respuestas culturales y su control varían de acuerdo a las poblaciones afectadas. Para profundizar en este tema se estudiaron 25 individuos que conservaron abundante cabellera, donde se cuantificó la presencia, ausencia y densidad de pediculosis en un área de 2 × $2 \mathrm{~cm}$ de cabello. De esta muestra, el 88\% (22/25) fue positiva para pediculosis, con una densidad promedio de 4,4 liendres. Cabe señalar que uno de los individuos analizados tuvo una densidad de 30 liendres en un área de 2 x $2 \mathrm{~cm}$, presentando la mayor cantidad de liendres del total de individuos estudiados. A la luz de esta evidencia, se discuten las prácticas culturales asociadas a la pediculosis, y si la considerable cantidad de especímenes observados habría sido utilizada como tributo durante el periodo Inca, cuando no existía otra forma de contribuir al Estado. Se concluye que la pediculosis fue endémica y que su tolerancia cultural y cosmovisión contribuyó a su prevalencia en el periodo Tardío.
\end{abstract}

Palabras claves: pediculosis, ectoparásitos, prácticas culturales, tributos, Inca.

\begin{abstract}
In this work, we studied the prevalence of pediculosis in the Inca site, Camarones 9 (northern Chile), to contrast with descriptions contained in the chronicles and what has been observed in the bioarchaeological record. The head lice is a frequent ectoparasitosis, but the cultural responses and its control vary according to the affected populations. To delve into this topic, we studied 25 mummies with abundant hair, quantifying the presence, absence, and density of pediculosis in an area of hair measuring $2 \times 2 \mathrm{~cm}$. About $88 \%$ (22/25) of the mummies were found positive for head lice, with an average density of 4.4 nits per $2 \times 2 \mathrm{~cm}$. It should be noted that one of the individuals analyzed had a density of 30 nits in the same $2 \times 2 \mathrm{~cm}$ area, presenting the highest amount from the total number of individuals studied. In the light of this evidence, the cultural practices associated with pediculosis are discussed, as well as whether the considerable number of specimens observed would have been used as tribute during the Inca period when there was no other way to contribute to the state. It is concluded that pediculosis was endemic and that its cultural tolerance and worldview contributed to its prevalence during the Late period.
\end{abstract}

Key words: Head lice, ectoparasites, cultural practices, tributes, Inca.

La pediculosis de la cabeza es una ectoparasitosis provocada por Pediculus humanus capitis ( $P$. capitis), insecto comúnmente conocido como piojo, que se caracteriza por ser hematófago y parasitar exclusivamente al ser humano (Devera 2012; De Villalobos et al. 1998). P. capitis se transmite por contacto directo cabeza-cabeza, y el cabello largo facilita su ciclo reproductivo. Por otro lado, su alta tasa de reproducción dificulta su erradicación, ya que una hembra puede depositar hasta 100 huevos/liendres durante su vida, propagándose de forma endémica y causando malestares físicos y emocionales como picazón e irritabilidad en los individuos afectados (Heukelbach y Walton 2005; Lehane 2005).

\footnotetext{
Instituto de Alta investigación, Universidad de Tarapacá, Arica, Chile. barriazaarica@gmail.com; natalia.aravena.sanchez@gmail.com

${ }^{2 \dagger}$ Departamento de Biología, Universidad de Tarapacá, Arica, Chile.

3 Departamento de Antropología, Universidad de Tarapacá, Arica, Chile. vivien.standen@ gmail.com
} 
Debido a la gran resistencia y perdurabilidad que le brinda su exoesqueleto quitinoso, el piojo y sus huevos se conservan íntegramente en el registro arqueológico. Gracias a esto, su estudio ha servido de base para determinar su coevolución con los primates, patrones de migración de las antiguas poblaciones y para establecer antecedentes epidemiológicos (Ashfaq et al. 2015; Raoult et al. 2008; Reed et al. 2004; Weiss 2009).

El registro bioarqueológico de las Américas indica que la pediculosis presenta una notable antigüedad. En Brasil, Araújo et al. (2000) hallaron liendres en un sitio de 10.000 años de antigüedad, mientras que en Chile, Rivera et al. (2008) y Arriaza et al. (2013) registraron pediculosis en el cabello de momias pertenecientes a la Cultura Chinchorro (ca. 3050-1050 AC). Otras investigaciones como la realizada por Reinhard y Buikstra (2003), evidenciaron pediculosis en momias de la Cultura Chiribaya del sur del Perú (1000-1250 DC) y en Arica, Arriaza, Olivares et al. (2014) y Arriaza, Standen et al. (2014) observaron piojos y liendres en peines de los sitios AZ-71 y AZ71a del periodo Medio (500-1000 DC); CAM-8 y PLM 4 del Intermedio Tardío (1000-1450 DC) y PLM-6 del periodo Tardío (1600 DC).

Un aspecto singular de los estudios de pediculosis es la existencia de algunos individuos con alta carga parasitaria. Lo anterior es descrito por Arriaza et al. (2012), quienes analizan el caso de un adulto de sexo masculino perteneciente a la Cultura Maytas-Chiribaya en Arica (760 DC), el cual presentaba un máximo de 16 liendres en un área de $2 \times 2 \mathrm{~cm}$ de su cabello. Esto nos lleva a reflexionar sobre las respuestas culturales y la aceptación de la pediculosis en el pasado, ya que esta varía ampliamente entre poblaciones. Así, mientras que en la actualidad algunas comunidades presentan una aversión total a ella (Juárez et al. 2017; López et al. 2017), otras como los uros, por ejemplo, no perciben la pediculosis como un gran problema (Souffez 1985). Esta respuesta cultural es incluso más intrigante si consideramos que existe registro de poblaciones prehispánicas (Fry 1976; Reinhard 1990) y de diversas partes del mundo que los ingerían (Cieza de León 2005 [1553]:96; Malinowski 1975:247, 251; Páramo 2020).

La pediculosis en la antigüedad también ha sido mencionada por los cronistas, lo que nos hace considerar la relación entre piojos y personas como un amplio caleidoscopio de respuestas culturales. En este sentido, Cieza de León señala sobre los indígenas Pastos: "Y así ellos como todos los demás que se han pasado son tan poco asquerosos, que cuando se espulgan se comen los piojos como si fuesen piñones" (Cieza de León 2005 [1553]:96).

A esta declaración se suma:

Pues, es cosa muy notoria y entendida, ningún pueblo de la sierra, ni valle de los llanos dejó de pagar el tributo de derrama que le era impuesto por los que para ello tenían cargo, y aun tal provincia hubo, que diciendo los naturales no tener con qué pagar tributo, les mandó el rey que cada persona de toda ella fuese obligado de le dar cada cuatro meses un cañuto algo grande lleno de piojos vivos, lo cual era industria del Inga para imponerlos $\mathrm{y}$ avisarlos en el saber tributar $\mathrm{y}$ contribuir; y así, sabemos que pagaron tributo de piojos algunos días hasta que, habiéndoles mandado dar ganado, procuraron de lo criar y hacer ropas y buscar con qué tributar para el tiempo de adelante (Cieza de León 2005 [1553]:336-337).

Por otro lado, Garcilaso de la Vega aunque no menciona que las poblaciones del periodo Inca estén comiendo sus piojos, sí concuerda con la aseveración de Cieza de León respecto al pago de impuestos, señalando:

Otra manera de tributo daban los impedidos que llamamos pobres, y era que de tantos a tantos días eran obligados a dar a los gobernadores de sus pueblos ciertos cañutos de piojos. Dicen que los Incas pedían aquel tributo porque nadie (fuera de los libres de tributo) se ausentase de pagar pecho, por pobre que fuese, y que a éstos se lo pedían de piojos, porque, como pobres impedidos, no podían hacer servicio personal, que era el tributo que todos pagaban. Pero también decían que la principal intención de los Incas para pedir aquel tributo era celo amoroso de los pobres impedidos, por obligarles a que se despiojasen y limpiasen, porque, como gente desastrada, no pereciesen 
comidos de piojos. Por este celo que en toda cosa tenían los Reyes, les llamaban amadores de pobres. Los decuriones de a diez (que en su lugar dijimos) tenían cargo de hacer pagar este tributo (Garcilaso de la Vega 1985 [1609]:225).

Sobre la base de lo anterior y considerando la excelente preservación de los cabellos de las inhumaciones del sitio Camarones 9 (CAM-9), asociado al periodo Inca en el norte de Chile, queremos indagar hasta qué punto estas poblaciones estaban infestadas por pediculosis y debatir su importancia.

\section{Materiales y Métodos}

\section{Antecedentes arqueológicos}

Se estudiaron restos bioarqueológicos momificados y osamentas provenientes del sitio CAM-9 que se conservan en el Museo Arqueológico San Miguel de Azapa (MASMA) de la Universidad de Tarapacá, en Arica, Chile. El sitio se encuentra ubicado a $100 \mathrm{~km}$ al sur de Arica en la desembocadura del Valle de Camarones (Figura 1) y corresponde a un cementerio asociado al periodo Incaico (ca.
1370-1450 DC) (Carmona 2006; Muñoz 1989) con algunos componentes del periodo Colonial (ca. 1600 DC) (Catalán 2008).

En el valle y desembocadura del Río Camarones, Muñoz (1989) y Schiappacasse y Niemeyer (1989) plantean la coexistencia de dos grupos de poblaciones, una dedicada a la pesca y otra a la agricultura. que dependían entre sí políticamente y que terminan siendo sometidas por grupos altiplánicos previamente absorbidos por el Imperio inca. Al respecto, CAM-9 es una población con una economía mixta de caza, pesca, recolección y agricultura, que exhibe cuerpos con momificación natural, acuclillados, enterrados en posición fetal y envueltos de pies a cabeza en mantas monocromas o bicromas formando un fardo funerario (Muñoz 1989; Ulloa et al. 2000). Los cuerpos en su mayoría tienen el cabello largo, el que entramaron con un conjunto de pequeñas trenzas tanto en la parte posterior como a cada lado de la cabeza (Figura 2), apreciándose además ornamentaciones cefálicas en la parte externa de los fardos funerarios.

Camarones 9 fue excavado en los inicios de la década de 1960 por el equipo del Museo Regional de Arica, pero muchos de los fardos quedaron in situ. Posteriormente, en 1985, Percy Dauelsberg y Vivien Standen, de la Universidad de Tarapacá,

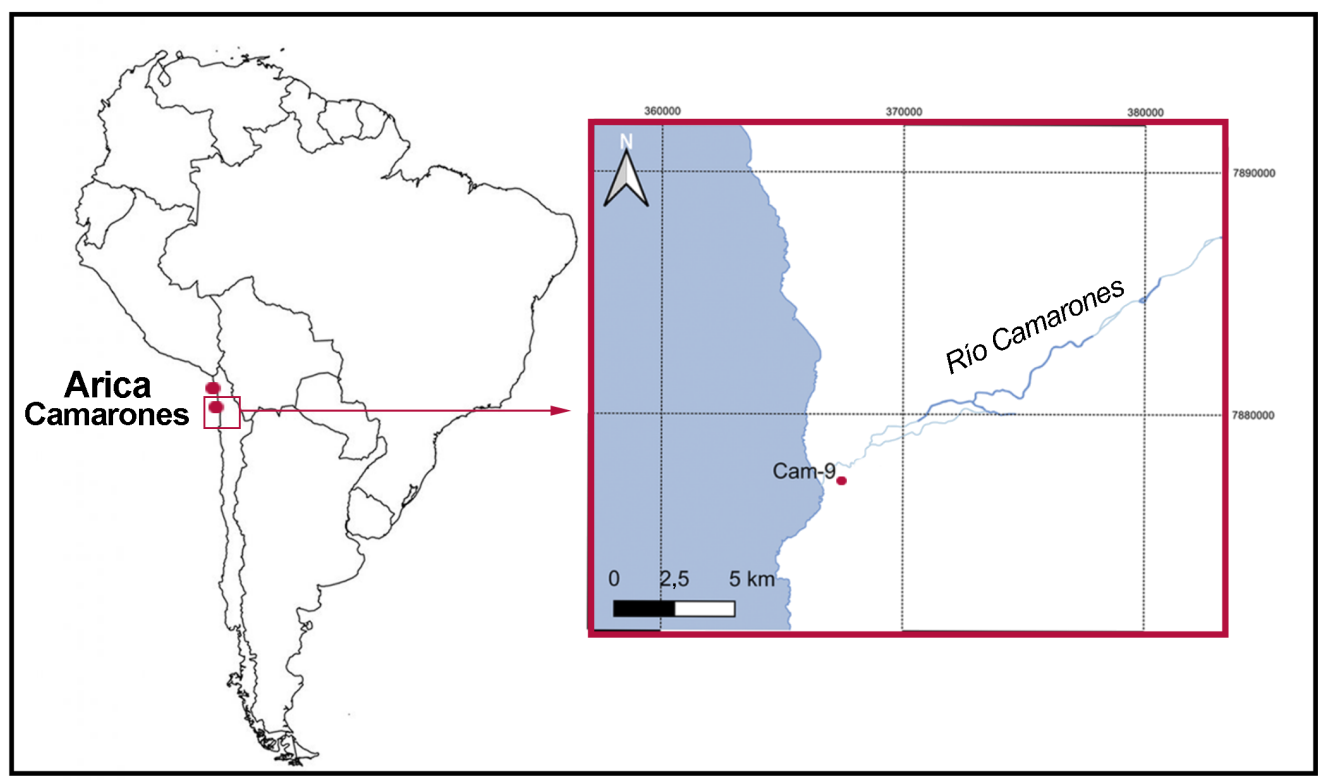

Figura 1. Localización del sitio arqueológico Camarones 9.

Location of Camarones 9 archaeological site. 


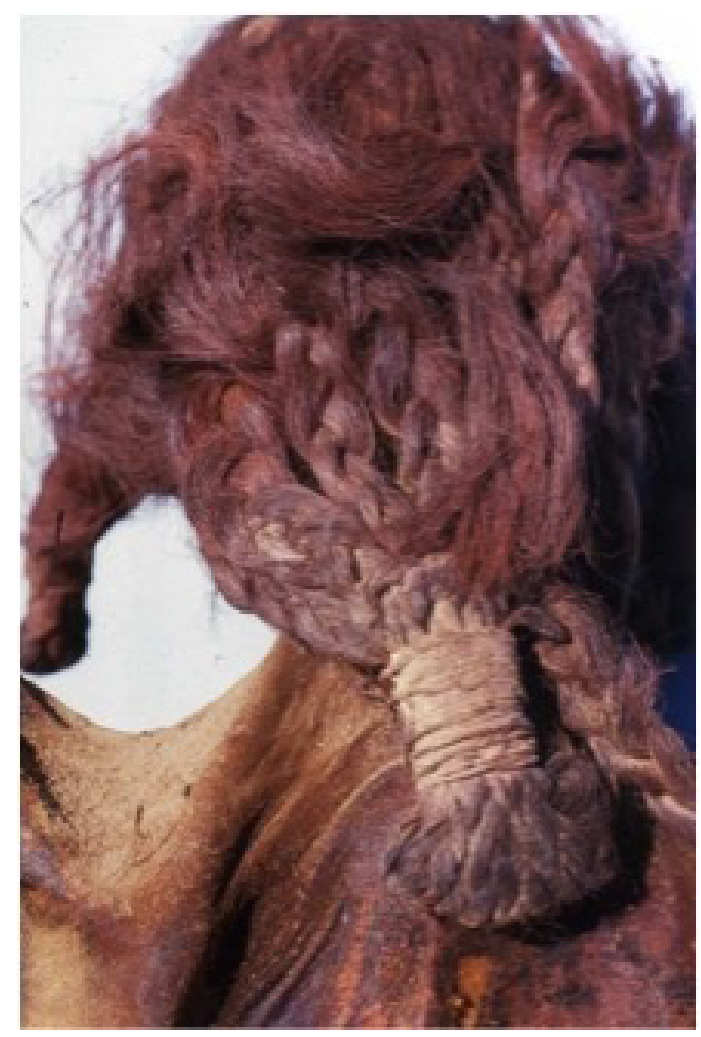

Figura 2. Individuo masculino de la población incaica de Camarones 9 con múltiples trenzas. Vista posterior.

Male individual from Camarones 9 Inca site showing multiple braids. Posterior view.

retomaron la excavación del sitio, localizando 45 tumbas con un patrón funerario en fosas de entierros individuales con abundantes ofrendas como anzuelos, remos en miniatura, plomadas, torteros y alfarería con formas de aríbalos, entre otros (Catalán 2008; Muñoz 1989; Schiappacasse y Niemeyer 1989; Ulloa et al. 2000). A fines de la década de los ochenta, estos fardos funerarios fueron abiertos y se realizaron autopsias a los cuerpos con el objetivo de analizar las enfermedades precolombinas, lo que se tradujo en publicaciones como el estudio de arsenicismo crónico (Figueroa et al. 1988) y mal de Chagas (Aufderheide et al. 2005). Los esqueletos de este sitio también han sido utilizados para otros estudios, como el de dieta (King et al. 2018) y anomalías óseas como la espina bífida (Silva-Pinto et al. 2010). Por último, basados en la alta presencia de lesiones cutáneas y arsénico acumulado en diversos tejidos del cuerpo de los individuos de CAM-9, Arriaza et al. (2018) postulan que este sitio podría corresponder a un cementerio de poblaciones foráneas (mitimaes). Señalan que al ser comunidades relocalizadas y no previamente expuestas al arsénico, la toxicidad del hidroarsenicismo habría afectado significativamente la salud y capacidad productiva de los individuos de CAM-9.

\section{Muestra estudiada}

Si bien en la literatura se describen 45 individuos en CAM-9, en este estudio solo se revisó una muestra de 25 individuos disponibles en el MASMA para examinación detallada, consultándose los registros de las autopsias para complementar y/o verificar los datos bioarqueológicos cuando fue posible. La mayoría de los casos estudiados, que fueron autopsiados en los años ochenta estaban completos (20/25), correspondían a individuos momificados naturalmente, cuyos huesos y tejidos blandos fueron preservados después de la autopsia. En aquellos casos en los que la disección fue completa, el cuero cabelludo fue removido manteniendo el cabello adherido a este $(n=22)$. En tres casos las cabezas no estaban diseccionadas y conservaban sus cabellos (Tabla 1). Los esqueletos preservados de CAM-9 (post autopsia) permitieron su estudio bioantropólogico.

La estimación del sexo en las muestras esqueletales (asociadas a cuero cabelludo) fue realizada por los autores utilizando los criterios estándares del dimorfismo de la pelvis, en particular las características morfológicas de la rama púbica y escotadura ciática mayor (Bass 1987; Coleman 1969), además de las características craneanas (Bass 1987; Campillo y Subirà 2004; White y Folkens 1991). En relación con la edad, los individuos fueron subdivididos en dos categorías: subadultos y adultos. En la primera categoría se utilizó el desarrollo dental (AlQahtani et al. 2010; Ubelaker 1978), la fusión de las epífisis y de los centros de osificación secundaria (Scheuer y Black 2000) y en la segunda, la morfología de la sínfisis púbica y de la superficie auricular (Brooks y Suchey 1990; Lovejoy et al. 1985).

\section{Procedimientos para la búsqueda de pediculosis}

Para la pesquisa de pediculosis, en el MASMA se inspeccionaron tanto los cabellos directamente en las cabezas $(n=3)$ como el cuero cabelludo producto de las autopsias $(\mathrm{n}=22)$. Durante el análisis, los restos bioarqueológicos fueron depositados en una hoja blanca y limpia de papel sin ácido, con el objetivo de recoger 
Tabla 1. Resultados del análisis de pediculosis de los 25 individuos inca.

Results of Pediculosis capitis analysis on 25 Inca individuals.

\begin{tabular}{|c|c|c|c|c|c|c|c|c|c|c|c|c|}
\hline \multirow[b]{3}{*}{ Cementerio } & \multirow[b]{3}{*}{ Sexo } & \multicolumn{2}{|c|}{ Antecedentes } & \multicolumn{3}{|c|}{$\begin{array}{c}\text { Análisis macroscópico en } \\
\text { MASMA } \\
\text { (análisis directo en el } \\
\text { cabello de las momias) }\end{array}$} & \multicolumn{6}{|c|}{$\begin{array}{l}\text { Análisis microscópico en Laboratorio Bioarqueología }{ }^{2} \\
\text { (liendres y piojos en cabeza y cuero cabelludo de autopsia) }\end{array}$} \\
\hline & & \multirow[b]{2}{*}{ Edad (años) } & \multirow[b]{2}{*}{ Categoría de edad } & \multirow[b]{2}{*}{$\begin{array}{l}\text { Segmento anatómico } \\
\text { analizado }^{3}\end{array}$} & \multirow{2}{*}{$\begin{array}{l}\text { Promedio } \\
\text { de liendres } \\
\text { en área de } \\
2 \times 2 \mathrm{~cm}^{4}\end{array}$} & \multirow[b]{2}{*}{$\begin{array}{l}\mathrm{N}^{\circ} \text { de piojos } \\
\text { contados }\end{array}$} & \multicolumn{3}{|c|}{$\begin{array}{l}\text { Detalle del análisis directo de } \\
\text { piojos (cabello) }\end{array}$} & \multirow{2}{*}{$\begin{array}{l}\mathrm{N}^{\circ} \text { de } \\
\text { liendres en } \\
\text { muestra de } \\
\text { cabello }\end{array}$} & \multirow{2}{*}{$\begin{array}{l}\quad \mathrm{N}^{\circ} \text { de } \\
\text { liendres en } \\
\text { muestra de } \\
\text { sedimento }\end{array}$} & \multirow{2}{*}{$\begin{array}{c}\mathrm{N}^{\circ} \text { de } \\
\text { piojos en } \\
\text { muestra de } \\
\text { sedimento }\end{array}$} \\
\hline & & & & & & & $\stackrel{\widetilde{m}}{\stackrel{\Xi}{\Xi}}$ & 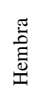 & 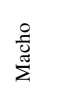 & & & \\
\hline CAM-9 T1 & M & $45-50$ & Adulto & C. cabelludo & 4 & 3 & 0 & 2 & 1 & 10 & 2 & 0 \\
\hline CAM-9 T2 & $\mathrm{F}$ & $25-30$ & Adulto & C. cabelludo & 4 & 2 & 0 & 1 & 1 & 9 & 0 & 1 \\
\hline CAM-9 T4 & M & $3-4$ & Subadulto & C. cabelludo & 0,7 & 1 & 1 & 0 & 0 & 3 & 0 & 0 \\
\hline CAM-9 T8 & M & $40-45$ & Adulto & C. cabelludo & 5 & 2 & 0 & 2 & 0 & 1 & 0 & 0 \\
\hline CAM-9 T9 & M & $4-5$ & Subadulto & C. cabelludo & 10,3 & 7 & 3 & 3 & 1 & 14 & 0 & 0 \\
\hline CAM-9 T10 & M & $12-15$ & Subadulto & C. cabelludo & 10 & 8 & 4 & 2 & 2 & 17 & 1 & 1 \\
\hline CAM-9 T11 & M & $6-8$ & Subadulto & C. cabelludo & 0 & 0 & 0 & 0 & 0 & 0 & 0 & 0 \\
\hline CAM-9 T12 & $\mathrm{F}$ & $35-40$ & Adulto & C. cabelludo & 1,3 & 0 & 0 & 0 & 0 & 5 & 0 & 0 \\
\hline CAM-9 T13 & M & $40-45$ & Adulto & C. cabelludo & 0,3 & 0 & 0 & 0 & 0 & 1 & 0 & 0 \\
\hline CAM-9 T14 & M & $20-25$ & Adulto & Cabeza & 2 & 0 & 0 & 0 & 0 & 9 & 1 & 0 \\
\hline CAM-9 T16 & $\mathrm{F}$ & $30-35$ & Adulto & C. cabelludo & 2 & 3 & 2 & 0 & 1 & 4 & 0 & 0 \\
\hline CAM-9 T23 & M & $14-16$ & Subadulto & C. cabelludo & 2,3 & 0 & 0 & 0 & 0 & 6 & 1 & 0 \\
\hline CAM-9 T29 & $\mathrm{F}$ & $18-20$ & Adulto & C. cabelludo & 30,7 & 66 & 39 & 14 & 13 & 83 & 33 & 35 \\
\hline CAM-9 T32C1 & Indet. & $3-4$ & Subadulto & C. cabelludo & 0 & 0 & 0 & 0 & 0 & 0 & 0 & 0 \\
\hline CAM-9 T33 & M & $40-45$ & Adulto & C. cabelludo & 0 & 0 & 0 & 0 & 0 & 0 & 0 & 0 \\
\hline CAM-9 T35 & M & $30-35$ & Adulto & C. cabelludo & 5 & 1 & 1 & 0 & 0 & 9 & 1 & 0 \\
\hline CAM-9 T36 & M & 15 & Adulto & C. cabelludo & 1,7 & 2 & 0 & 1 & 1 & 5 & 0 & 0 \\
\hline CAM-9 T37 & M & $35-40$ & Adulto & C. cabelludo & 1 & 0 & 0 & 0 & 0 & 3 & 0 & 0 \\
\hline CAM-9 T38 & M & $18-20$ & Adulto & C. cabelludo & 3,7 & 2 & 1 & 1 & 0 & 7 & 0 & 1 \\
\hline CAM-9 T38A & $\mathrm{F}$ & $17-20$ & Adulto & Cabeza & 10 & 4 & 2 & 1 & 1 & 36 & 0 & 0 \\
\hline CAM-9 T39 & M & $20-25$ & Adulto & Cabeza & 1 & 0 & 0 & 0 & 0 & 2 & 0 & 1 \\
\hline CAM-9 T45 & M & $40-45$ & Adulto & C. cabelludo & 8 & 0 & 0 & 0 & 0 & 12 & 4 & 1 \\
\hline CAM-9 T49 & M & Niño & Subadulto & C. cabelludo & 3 & 5 & 3 & 0 & 2 & 15 & 0 & 0 \\
\hline CAM-9 T52 & Indet. & Lactante & Subadulto & C. cabelludo & 3,3 & 0 & 0 & 0 & 0 & 23 & 3 & 0 \\
\hline \multirow[t]{3}{*}{ CAM-9 T54BAC1 } & Indet. & $7-8$ & Subadulto & C. cabelludo & 2,3 & 0 & 0 & 0 & 0 & 3 & 1 & 0 \\
\hline & & & Promedio & -- & 4,4 & 4,2 & 2,2 & 1,1 & 0,9 & 11,1 & 1,9 & 1,6 \\
\hline & & & Total & -- & -- & 106 & 56 & 27 & 23 & 277 & 47 & 40 \\
\hline
\end{tabular}

${ }^{1}$ Análisis macroscópico realizado en el Laboratorio de Antropología Física del Museo Arqueológico San Miguel de Azapa (MASMA).

${ }^{2}$ Análisis de microscopía óptica realizado en el Laboratorio de Bioarqueología del Instituto de Alta Investigación de la Universidad de Tarapacá.

${ }^{3}$ Segmento anatómico (C. cabelludo): Cuero cabelludo extraído durante la autopsia. Este segmento corresponde a cabello adherido a cuero cabelludo, no es posible identificar las zonas temporal y occipital.

${ }^{4} 2 \times 2 \mathrm{~cm}$ : Promedio total de liendres observadas en la zona temporal, occipital y/o en cabello suelto. 
todos los sedimentos que pudieran desprenderse. Posteriormente, siguiendo la metodología de Reinhard y Buikstra (2003) y Arriaza et al. (2012, 2013), se cuantificaron a ojo desnudo las liendres y piojos con la ayuda de una cartilla con un cuadrado calado de 2 x $2 \mathrm{~cm}$, la cual se aplicó en la zona temporal y occipital, levantando el cabello. Dicha observación se realizó en la zona cercana a la raíz del cabello, teniendo presente que en este sector el piojo coloca sus huevos y se alimenta varias veces en el día. Cabe señalar que las hembras depositan los huevos en el cabello, cerca del cuero cabelludo debido a que esta área presenta condiciones óptimas de temperatura y humedad para el desarrollo de la liendre, por lo que esta zona contiene los últimos eventos reproductivos del piojo (Burgess 2010). Basados en lo expuesto anteriormente, se realizaron tres recuentos en el cuero cabelludo de las zonas temporal y occipital, con la finalidad de calcular la prevalencia de pediculosis, la densidad máxima de liendres en 2 x $2 \mathrm{~cm}$ y el promedio de piojos observados.

\section{Muestreo}

En el MASMA se tomaron muestras de cabello con posibles liendres de las zonas anatómicas estudiadas, se recolectaron los piojos observados y se muestreó todo el sedimento que se desprendió de la cabeza o cuero cabelludo durante el análisis de los cuerpos.

\section{Procedimientos para el análisis microscópico}

Las muestras recolectadas fueron analizadas en el Laboratorio de Bioarqueología del Instituto de Alta Investigación de la Universidad de Tarapacá (IAI), utilizando un microscopio Olympus BX-41 y un microscopio electrónico de barrido Zeiss EVO LS-10. Se trabajó sobre la base de tres objetivos: primero, confirmar la presencia de liendres y así descartar que interferentes como concreciones de arena o sedimentos fueran confundidos con estos especímenes; segundo, para determinar el estadio de las liendres recolectadas (operculadas con el embrión dentro y cerradas, o decorticadas sin el embrión) y piojos (ninfas, hembras o machos); tercero, para caracterizar métricamente los especímenes. Solo aquellas liendres que estaban completas (sin fragmentar) y los piojos que contaran con cabeza, tórax y abdomen (espécimen completo) fueron considerados para el análisis morfométrico.

\section{Resultados}

Del total de 25 individuos analizados, solo tres fueron negativos para pediculosis, obteniéndose una prevalencia de $88 \%$ (22/25) (Tabla 1). Del total de individuos analizados, 17 fueron masculinos, cinco femeninos y tres indeterminados, mientras que con base en la edad, nueve correspondieron a subadultos y 16 a adultos (mayores de 18 años). Al estudiar la pediculosis según el sexo, los individuos masculinos presentaron un $88,2 \%$ (15/17) y los femeninos un $100 \%$ (5/5) de positividad, mientras que el $66,7 \%(2 / 3)$ de los individuos de sexo indeterminado fue positivo al análisis.

El análisis de pediculosis de acuerdo a la edad refleja que el 77,8\% (7/9) de los subadultos estudiados fueron positivos a la pesquisa, mientras que el $93,8 \%(15 / 16)$ de los adultos tuvieron pediculosis, diferencia que no es significativa $\left(X^{2}=1,39 ; p=0,24\right)$.

En lo que respecta a la densidad de liendres, se obtuvo un promedio de 4,4 especímenes en un área de $2 \times 2 \mathrm{~cm}$ de cabello analizado. En este punto destaca el caso de CAM-9 T29, un adulto femenino, que presentó una densidad de 30 liendres en el área de $2 \times 2 \mathrm{~cm}$. Otros tres individuos de diferente sexo y edad (CAM-9 T9, CAM-9 T10 y CAM-9 T38A) presentaron una densidad promedio de 10 liendres en el área de $2 \times 2 \mathrm{~cm}$, valores que incrementaron el promedio general de liendres para el sitio en cuestión (Tabla 1, Figura 3).

En referencia al análisis microscópico de las muestras recolectadas en el MASMA, se contabilizaron

Figura 3. Dos liendres eclosionadas adherıdas al cabello del individuo Camarones 9 T29. Visualización en microscopía óptica. 
324 liendres, de las cuales 82 estaban fragmentadas, 194 decorticadas (eclosionadas) y 48 operculadas (sin eclosionar), obteniéndose que el valor promedio del largo máximo del huevo fue de $858 \mu \mathrm{m}$ para las liendres decorticadas y $920 \mu \mathrm{m}$ en las liendres operculadas (Tabla 2).

En cuanto a los piojos observados en las muestras recolectadas en el MASMA, se contabilizaron 106 especímenes, de los cuales 56 correspondieron a ninfas, 27 a hembras y 23 a machos. De este total solo 12 hembras y 19 machos cumplieron con el criterio para analizarse métricamente en microscopía óptica (Figuras 4A y 4B). Este análisis reveló que las hembras eran significativamente más grandes que los machos, 2814 versus $2362 \mu \mathrm{m}$ (Tabla 2), $(\mathrm{t}=2,09 ; \mathrm{p}<0,001)$.

\section{Discusión y Comentarios}

La presencia de Pediculus capitis en los individuos estudiados del sitio CAM-9 fue alta, obteniéndose un $88 \%(22 / 25)$ de muestras positivas al análisis. inspeccionar la cabeza y cuero cabelludo, los cuales normalmente no son estudiados, estos resultaron ser especialmente importantes en los individuos altamente infestados. Por último, destacamos que se observó una excelente concordancia entre el análisis visual a ojo desnudo (en MASMA) y el microscópico (en laboratorio), en relación a la prevalencia de pediculosis.

Según lo descrito anteriormente, la pediculosis fue altamente prevalente en diferentes periodos de las poblaciones andinas. En el caso del periodo Tardío, y considerando el argumento de los cronistas, se podría discutir la pediculosis de CAM-9 bajo cuatro perspectivas: (1) el pago de tributo, (2) higiene, (3) pobreza y (4) idiosincracia cultural. En el primer enfoque, argumentamos que el trasfondo del pago de tributo con piojos fue un esfuerzo del Inca para señalar que todos los súbditos debían aportar al Imperio y pagar los impuestos. Hasta donde sabemos, la cultura material de CAM-9 no reporta cañutos o contenedores con piojos, pero sí peines para el despioje. En relación al segundo punto, la remoción de liendres y piojos requiere de la ayuda de un tercero, a menudo una

Tabla 2. Resumen de los datos métricos de la observación microscópica de Pediculus humanus capitis (en $\mu$ ). Synthesis of metric and microscopic observations of the Pediculus humanus capitis specimens (in $\mu \mathrm{m}$ ).

\begin{tabular}{ccccccccc}
\hline \multicolumn{2}{c}{ Tipo de muestra } & Variable & Promedio & $\mathrm{n}$ & $\mathrm{DE}$ & \multicolumn{2}{c}{ Valor mínimo } & \multicolumn{2}{c}{ Valor máximo } \\
\hline \multirow{5}{*}{ Liendre } & Operculada & Largo máximo & 920 & 48 & 57 & 800 & 1.079 \\
\cline { 2 - 8 } & Operculada & Ancho máximo & 393 & 31 & 35 & 312 & 469 \\
\cline { 2 - 8 } & Decorticada & Largo máximo & 858 & 194 & 55 & 679 & 968 \\
\cline { 2 - 8 } & Decorticada & Ancho máximo & 392 & 133 & 30 & 315 & 471 \\
\hline \multirow{3}{*}{ Piojo } & Hembra & Largo máximo & 2.814 & 12 & 182 & 2.544 & 7.172 \\
\cline { 2 - 8 } & Hembra & Ancho máximo & 913 & 12 & 123 & 749 & 2.658 \\
\cline { 2 - 8 } & Macho & Largo máximo & 2.362 & 19 & 226 & 2.107 & 615 & 797 \\
\hline
\end{tabular}

Este valor es más del doble de lo reportado por Reinhard y Buikstra (2003) en momias de la Cultura Chiribaya de Perú, que presentan un $34,7 \%$ (34/98) de prevalencia de pediculosis. Sin embargo, los resultados observados en CAM-9 son comparables con los de Arriaza et al. (2013) para los grupos del periodo Arcaico del norte de Chile (Chinchorro), que presentaron un 79,4\% (50/63) de positividad. Además, coinciden con lo reportado por Rivera et al. (2008), quienes obtuvieron un $85,7 \%$ (6/7) de pediculosis en individuos pertenecientes a CAM15-D, sitio arqueológico asociado a la Cultura Chinchorro.

En lo que se refiere a la observación de pediculosis en las muestras de sedimento que se desprendieron al persona cercana (pariente, amigo o pareja), por lo que la solución más eficaz hubiera sido que el Inca ordenase cortar los cabellos de sus súbditos infestados con pediculosis. Al contrario, la población mantenía su cabello largo con mucho esmero y lo trenzaban de múltiples maneras. En la prehistoria regional de Arica, desde el periodo Medio hacia delante, las mujeres por lo general llevaban largas trenzas, una a cada lado; y los hombres, múltiples trenzados (Arriaza et al. 1986), lo que seguramente facilitaba la reproducción y propagación de la pediculosis. Considerando que los cánones de belleza están imbricados en la cultura, en esos tiempos se prefería una cabellera larga y con ectoparásitos antes que raparse; así la 


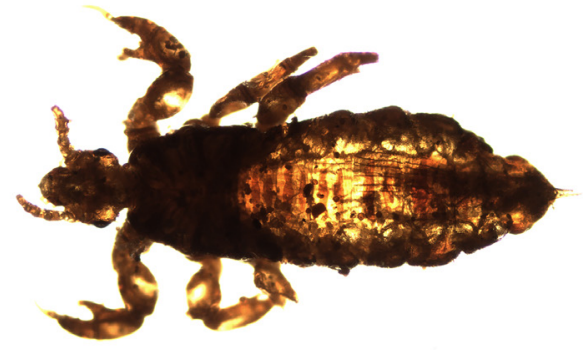

A

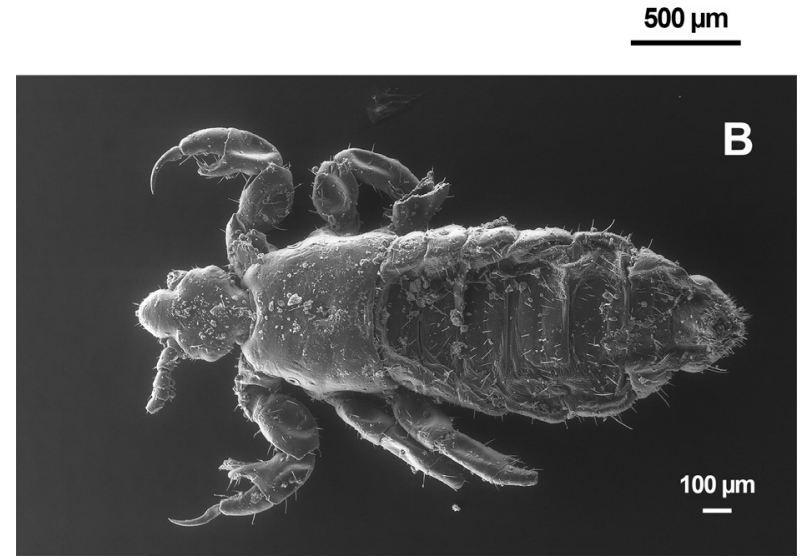

Figura 4. Espécimen masculino de Pediculus humanus capitis extraído del individuo Camarones 9 T1. (A) Visualización en microscopía óptica (vista ventral); (B) visualización en microscopía electrónica de barrido (vista dorsal).

Male specimen of Pediculus humanus capitis, sampled from Camarones 9 T1. (A) Optical microscopy (ventral view); (B) Scanning electron microscopy (dorsal view).

pediculosis se propagó como una pandemia endémica, milenaria, tolerada y mantenida. Sobre el uso de una larga cabellera, el cronista Cobo señala: "Los días que duraban los lloros hasta el del entierro, los que asistían a ellos se cubrían las cabezas con sus mantas; y muchas de las mujeres de los señores, en la muerte dellos, para mayor demostración de tristeza, se cortaban el cabello" Cobo (1964 [1653]:274); "Las mujeres y sirvientes se cortaron el cabello solamente como signo de luto, tristeza o desgracia". "Tienen puesta toda su honra en la cabellera en tanto grado, que la mayor afrenta que se les puede hacer es cortársela, y como tal les suele dar esta pena la Justicia a los que cometen delitos graves e infames" Cobo (1964 [1653]:237).

En relación con el tercer punto, hay numerosos estudios actuales que indican que aunque la pediculosis puede encontrarse en todos los estratos sociales, la pobreza y el hacinamiento pueden aumentar su prevalencia y agravar esta ectoparasitosis (Cazorla et al. 2007; Devera 2012; De Villalobos 1998; López 2008). En este sentido, aunque las poblaciones de
CAM-9 no evidencian rasgos de extrema pobreza o abandono personal que minimizara el despiojamiento, sí presentan algunos problemas de salud producto del hidroarsenicismo (Brito 2020; Figueroa et al. 1988).

En lo que se refiere al cuarto punto, algunas poblaciones presentan fobia a la pediculosis, mientras que otras la ven como un mal menor con el que se aprende a convivir. Tal es el caso del pueblo de los uros en el Lago Titicaca, que no se avergonzaban por el hecho de tenerlos (Souffez 1986). En el caso de CAM-9, ¿podría ser la tolerancia cultural el motivo por el cual algunos individuos presentan una alta densidad de liendres y piojos? Ciertamente la actitud sociocultural de tolerancia o rechazo frente a los ectoparásitos es un factor importante en la tasa de infestación y prevalencia comunitaria. También se debe considerar que los piojos presentan una alta tasa de reproducción y cerca del $10 \%$ de los individuos de una población contienen el mayor reservorio de parásitos, fenómeno que es conocido como distribución gregaria (Anderson 1993; Arriaza et al. 2013; Reinhard y Buisktra 2003), cualidad que los vuelve un foco 
de contagio. En nuestro estudio, los casos con una carga mayor de pediculosis varían entre el $4 \%$ (un individuo) y $16 \%$ (cuatro individuos), es decir, que en el caso más extremo aproximadamente uno de cada seis individuos contendría el mayor reservorio de este insecto, lo cual es relevante si consideramos que el cementerio CAM-9 es pequeño (45 inhumaciones). Así, destaca el caso de CAM-9 T29, en quien se registró una densidad de 30 liendres en un área de $2 \times 2 \mathrm{~cm}$, 66 piojos en el cabello y 35 piojos en el sedimento que se desprendió al momento de analizar su cabeza.

Entonces cabe preguntarse si hubo una intención de mantener los piojos, ya fuera porque no les molestaban y preferían los peinados complejos con un poco de picor antes que remover estos insectos, ¿o fueron los piojos parte importante del complejo entramado social, como lo señala Souffez (1985), del cuidado mutuo hombre-mujer y de lo íntimo? El despiojamiento implica un nivel de confianza, ya que la persona que es despiojada queda vulnerable por la posición física que asume al momento de remover las liendres y piojos.

Estimamos que efectivamente llevar el cabello largo en la prehistoria de Arica favoreció la alta prevalencia de pediculosis. Además, aunque el Inca cobraba impuestos de piojos, no creó una política en contra de los afectados por pediculosis. Por otra parte, si bien es cierto se contaba con peines para despiojarse, estos no fueron suficientemente efectivos para remover la totalidad de las pequeñas liendres, debido a la separación de sus dientes cuyo espesor mínimo equivale a medio milímetro (Arriaza, Standen et al. 2014). Por ende, también era necesario complementar el despiojamiento con una actividad manual larga y tediosa pero que permitía la interacción social.

Los estudios de coprolitos podrán contribuir al debate acerca de si los piojos fueron ingeridos, como ha sido reportado para otras poblaciones del mundo (Fry 1976; Malinowski 1975; Páramo 2020; Reinhard 1990) y queda abierta la interrogante de cuál fue el destino final de los piojos recolectados por el Inca. Por otro lado, tomando en cuenta su prevalencia (siete de cada ocho individuos fueron positivos) y su alta densidad en CAM-9, pareciera más bien que los piojos convergen hacia la tolerancia cultural idiosincrática, a lo sagrado y la fertilidad. Los piojos serían el símbolo de la riqueza y de la abundancia, como lo señala Souffez $(1985,1986)$ para las poblaciones andinas del siglo XVI y XVII. Si bien las referencias etnohistóricas están asociadas a otras zonas de los Andes, postulamos hipotéticamente, entonces, que la pediculosis en CAM-9 pudo haber sido deseable y los piojos vistos como parte de la fertilidad natural. Pequeños insectos que, a pesar de provocar malestar, comparten un espacio común, y que si bien se realizaba un despioje con peines especializados y también directamente con las manos, no se buscaba su eliminación total. En este aspecto, el Manuscrito de Huarochirí señala su relación con lo mítico cuando Cuniraya Wiracocha, hacedor del mundo, también podía ser representado como un hombre humilde y piojoso.

\section{Este Cuniraya Viracocha, en los tiempos más antiguos, anduvo, vago, tomando la apariencia de un hombre muy pobre; su yacolla (manto) y su cusma (túnica) hechas jirones. Algunos, que no lo conocían, murmuraban al verlo: "miserable piojoso", decían... (Ávila 1966 [¿1598?]:20).}

También se ha reportado, la huaca "piojosa" en Pacasmayo (en la costa norte de Perú), que acerca los piojos a lo sagrado (Souffez 1985, 1986). Más aún, es relevante que el Inca aceptase o pidiese un tributo de piojos. Este acto acercaba al súbdito tributante al Inca, porque despiojarse es un acto privado de cariño y cuidado. Además, el Inca era considerado una Divinidad, entonces también acercaba lo tributado (el piojo) a otra dimensión social, a lo sagrado y a lo divino, digno de ser cobrado como tributo. Hipotéticamente, en nuestra área de estudio es posible que los piojos también hayan formado parte del tributo, especialmente en aquellos individuos que presentaron una sobrecarga de este ectoparásito.

En el marco del simbolismo del piojo, si bien en nuestro folclore abundan las frases que le otorgan un aspecto negativo a la pediculosis como "los piojos son de los pobres", también hay frases de antípoda como "los niños que tienen abundancia de piojos serán ricos", el "contar con piojos anuncia riqueza", y "soñar con piojos significa que se recibirá dinero" (Plath 1956). Es sabido que en diferentes culturas los animales son tratados de forma diferenciada, y en algunos casos como animales sagrados (p.ej., vacas, monos, ratones, escarabajos, entre otros) (Espinosa 1996; Lira 1997; Monserrat 2013). Entonces, considerando la prevalencia extrema y lo señalado para el tiempo de los incas, sugerimos en concordancia con Souffez $(1985,1986)$ que los piojos pudieron llegar a ocupar un lugar especial dentro de la sacralidad andina y se 
les toleraba, o incluso se les consideraba como un bien tributable. Además, teniendo en cuenta su presencia desde épocas arcaicas, esta parasitosis fue endémica. Al respecto, Arriaza, Olivares et al. (2014:236) señalan:

\begin{abstract}
En la prehistoria, varios factores como el largo del cabello, los complejos peinados, el hacinamiento y la densidad de la población influían en la probabilidad de contraer piojos de la cabeza. A su vez, a pesar de que los peines eran instrumentos eficaces en la remoción de piojos, la prevalencia continuó siendo elevada. Planteamos que lo anterior se debe en parte a la alta tasa de reproducción de los piojos de la cabeza y al hecho de que los peinados se mantienen durante largos periodos de tiempo, por tanto el ciclo de vida del Pediculus humanus capitis no era alterado significativamente.
\end{abstract}

Actualmente, la pediculosis sigue estando presente en todo el mundo, afectando a diversas poblaciones sin discriminar entre grupos étnicos o clases sociales. Sin embargo, hoy en día en el mundo occidental presenta una aversión total a ella, generando un estigma social que la relaciona con suciedad y pobreza. Estas consideraciones contrastan radicalmente con las apreciaciones de antaño (Cieza de León 2005 [1553]; Souffez 1985), la de otros grupos étnicos actuales (Páramo 2020) y lo observado en CAM-9. Se concluye entonces que la pediculosis fue endémica y que su tolerancia cultural y cosmovisión contribuyó a su prevalencia en el periodo Tardío, presentando incluso una utilidad para el pago de impuestos en aquellos más desposeídos.

Agradecimientos: Proyecto de Tesis de Investigación para estudiantes de postgrado, Universidad de Tarapacá, 2016 (Código: 8760-16) y al Proyecto Fondecyt No 1100059 . Agradecemos a los evaluadores por sus comentarios y sugerencias constructivas. Dedicamos este trabajo a nuestro compañero y amigo Hipólito Núñez, Q.E.P.D. con quien compartimos gratas jornadas de investigación y colaboración.

\section{Referencias Citadas}

AlQahtani, S., M. Hector y H. Liversidge 2010. Brief Communication: The London atlas of human tooth development and eruption. American Journal of Physical Anthropology 142 (3):481-490.

Anderson, R.M. 1993. Epidemiology. En Modern Parasitology, 2nd ed., editado por E.F.G. Cox, pp. 75-116. Blackwell, Oxford.

Araújo, A., L. Ferreira, N. Guidon, N. Maues de Serra Freire, K Reinhard y K. Dittmar 2000. Ten thousand years of head lice infection. Parasitology Today 16 (7):269.

Arriaza, B., M. Allison, V. Standen, G. Focacci y J. Chacama 1986. Peinados precolombinos de Arica. Chungara Revista de Antropología Chilena 16-17:353-375.

Arriaza, B., D. Amarasiriwardena, V. Standen, J. Yáñez, J. Van Hoesen y L. Figueroa 2018. Living in poisoning environments: Invisible risks and human adaptation. Evolutionary Anthropology 27:188-196.

Arriaza, B., F. Olivares y V. Standen 2014. Peines para el control de la pediculosis en la prehistoria de Arica, Chile. En Avances recientes en la Bioarqueología Sudamericana, editado por L. Luna, C. Aranda y J. Suby, pp. 231-239. Grupo de Investigación en Bioarqueología, Buenos Aires.

Arriaza, B., N. Orellana, H. Barbosa, R. Menna-Barreto, A. Araújo y V. Standen 2012. Severe head lice infestation in an Andean mummy of Arica, Chile. The Journal of Parasitology 98 (2):433-436.

Arriaza, B., V. Standen, J. Heukelbach, V. Cassman y F. Olivares 2014. Peines para el despiojamiento en las antiguas poblaciones de Arica: rascando la evidencia. Chungara Revista de Antropología Chilena 46 (4):693-706.

Arriaza, B., V. Standen, K. Reinhard, A. Araújo, J. Heukelbach y K. Dittmar 2013. On head lice and social interaction in archaic Andean coastal populations. International Journal of Paleopathology 3:257-268.
Ashfaq, M., S. Prosser, S. Nasir, M. Masood, S Ratnasingham y P. Hebert 2015. High diversity and rapid diversification in the head louse, Pediculus humanus (Pediculidae: Phthiraptera). Scientific Reports 5:14188.

Aufderheide, A, W. Salo, M. Madden, J. Streitz, K. Dittmar, J. Buikstra, B. Arriaza y L.E. Wittmers 2005. Aspects of ingestion transmission of Chagas disease identified in mummies and their coprolites. Chungara Revista de Antropología Chilena 37 (1):85-90.

Ávila, F. de 1966 [¿1598?]. Dioses y Hombres de Huarochirí: Narración Quechua Recogida por Francisco de Ávila (¿1598?). Edición Bilingüe. Traducción de J. Arguedas y Estudio Bibliográfico de Pierre Duviols: Instituto de Estudios Peruanos y Museo Nacional de Historia, Lima.

Bass, W. 1987. Human Osteology: A Laboratory and Field Manual. Special Publication $N^{\circ} 2$. Missouri Archaeological Society, Columbia.

Brito, Y. 2020. Análisis Bioarqueológico de los Infantes Provenientes de los Sitios Camarones 8 y Camarones 9 y su Relación con la Contaminación Medioambiental. Memoria de Título, Departamento de Antropología, Universidad de Tarapacá, Arica.

Brooks, S. y J. Suchey 1990. Skeletal age determination based on the os pubis: A comparison of the Acsádi-Nemeskéri and SucheyBrooks methods. Human Evolution 5:227-238.

Burgess, I. 2010. Head lice biology. En Management and Control of Head Lice Infestations, editado por J. Heukelbach, pp. 23-31. UNI-MED Verlag AG, Bremen.

Campillo, D. y M.E. Subirà 2004. Antropología Física para Arqueólogos. Ariel Prehistoria, Barcelona.

Carmona, G. 2006. Los nuevos patrones formales y decorativos en las bolsas chuspa del área de Arica, bajo el dominio del Tawantinsuyu: una aproximación inicial. Actas del XVII Congreso 
Nacional de Arqueología Chilena, pp. 23-33. Sociedad Chilena de Arqueología, Valdivia.

Catalán, D. 2008. Reacciones Locales frente al Tawantinsuyu: Una Aproximación desde los Contextos Funerarios (El Período Tardío en los Valles Occidentales). Tesis para optar el grado de Magíster en Antropología, Universidad de Tarapacá-Universidad Católica del Norte, Arica.

Cazorla, D., A. Ruiz y M. Acosta 2007. Estudio clínico-epidemiológico sobre pediculosis capitis en escolares de Coro, estado Falcón, Venezuela. Investigación Clínica 48 (4):445-457.

Cieza de León, P. 2005 [1553]. Crónica del Perú y el Señorío de los Incas. Fundación Biblioteca Ayacucho, Caracas.

Cobo, B. 1964 [1653]. Historia del Nuevo Mundo. Estudio Preliminar del Padre Francisco Mateo de la Compañía de Jesús. Tomo II. Biblioteca de Autores Españoles desde la Formación del Lenguaje hasta Nuestros Días. Atlas, Madrid.

Coleman, W. 1969. Sex differences in the growth of the human bony pelvis. American Journal of Physical Anthropology 31:125-151.

Devera, R. 2012. Epidemiología de la pediculosis capitis en América latina. Saber Revista Multidisciplinaria del Consejo de Investigación de la Universidad de Oriente 24 (1):25-36.

De Villalobos, C., M. Ranalletta, A. Rodríguez y P. Sarmiento 1998. Pediculus humanus capitis bajo el microscopio electrónico. Archivos Argentinos de Dermatología 48:13-18.

Espinosa, G. 1996. Lari y Jamp'atu, ritual de lluvia y simbolismo andino en una escena de arte rupestre de Ariquilda 1. Norte de Chile. Chungara Revista de Antropología Chilena 28:133-157.

Figueroa, L., B. Razmilic, M. Allison y M. González 1988. Evidencia de arsenicismo crónico en momias del valle de Camarones. Región de Tarapacá, Chile. Chungara Revista de Antropología Chilena 21:33-42.

Fry, G. 1976. Analysis of prehistoric coprolites from Utah. Anthropological Papers 97:371-373.

Garcilaso de la Vega, Inca 1985 [1609]. Comentarios Reales de los Incas. Prólogo, edición y cronología de Aurelio Miró Quesada. Biblioteca Ayacucho, Caracas.

Heukelbach, J. y S. Walton 2005. Ectoparasitic infestations. Current Infectious Disease Reports 7:373-380.

Juárez, M., S. Atienza, M. Martín y G. Cañadas 2017. La estigmatización de los piojos. Medicina de Familia Andalucía 18 (1):61-70.

King, C., B. Arriaza, V. Standen, A. Millard, D. Gröcke, I. Muñoz y S. Halcrow 2018. Estudio isotópico del consumo de recursos marítimos y terrestres en la prehistoria del desierto de Atacama. Chungara Revista de Antropología Chilena 50 (3):369-396.

Lehane, M. 2005. The Biology of Blood-Sucking in Insects, second edition. Cambridge University Press, Cambridge.

Lira, C. 1997. El animal en la cosmovisión indígena. Aisthesis 30:125-142.

López, S. 2008. Guía Práctica para el Control de Piojos. Manuales de salud ambiental. Junta de Andalucía, Consejería de Salud, Andalucía.

López, D., A. Medina, S. Mosquera y L. Vásquez 2017. Actualización y perspectiva integrada de la pediculosis. Revista de la Asociación Colombiana de Dermatología y Cirugía Dermatológica 25 (1):38-48.

Lovejoy, C., R. Meindl, T. Pryzbeck y R. Mensforth 1985. Chronological metamorphosis of the auricular surface of the ilium:
A new method for the determination of adult skeletal age at death. American Journal of Physical Anthropology 68:15-28.

Malinowski, B. 1975. La Vida Sexual de los Salvajes del Noreste de la Melanesia. Descripción Etnográfica de las Relaciones Eróticas Conyugales y de la Vida de la Familia entre los Indígenas de las Trobriand (Nueva Guinea Británica). Tercera edición. Ediciones Morata S.A., Madrid.

Monserrat, V. 2013. Los artrópodos en la mitología, las creencias, la ciencia y el arte del antiguo Egipto. Boletín de la Sociedad Entomológica Aragonesa 52:373-437.

Muñoz, I. 1989. Perfil de la organización económica social en la desembocadura del río Camarones. Períodos Intermedio Tardío e Inca. Chungara Revista de Antropología Chilena 22:85-112.

Páramo, A. 2020. Eating lice. The Nasiona, humanize de other. https:// www.thenasiona.com/2020/07/17/eating-lice/(15 diciembre 2020)

Plath, O. 1956. Nuestro Folklore, los Insectos y otros Artrópodos Portadores de Enfermedades. Impr. de la Central de Abastecimiento del Servicio Nacional de Salud, Santiago.

Raoult, D., D. Reed, K. Dittmar, J. Kirchman, J. Rolain, S. Guillen y J. Light 2008. Molecular Identification of Lice from Pre-Columbian Mummies. The Journal of Infectious Diseases 197 (4):535-543.

Reed, D., V. Smith, S. Hammond, A. Rogers y D. Clayton 2004. Genetic analysis of lice supports direct contact between modern and archaic humans. PLoS Biology 2 (11):1973-1983.

Reinhard, K. 1990. Archaeoparasitology in North America. American Journal of Physical Anthropology 82:145-163.

Reinhard, K. y J. Buikstra 2003. Louse infestation of the Chiribaya culture, southern Peru: Variation in prevalence by age and sex. Memórias do Instituto Oswaldo Cruz 98 (1):173-179.

Rivera, M., K. Mumcuoglu, R. Matheny y D. Matheny 2008. Huevecillos de Anthropophthirus capitis en momias de la tradición Chinchorro, Camarones 15-D, norte de Chile. Chungara Revista de Antropología Chilena 40 (1):31-39.

Scheuer, L. y S. Black 2000. Developmental Juvenile Osteology. Academic Press, London.

Schiappacasse, V. y H. Niemeyer 1989. Avances y sugerencias para el conocimiento de la prehistoria tardía en la desembocadura del valle de Camarones (región de Tarapacá). Chungara Revista de Antropología Chilena 22:63-84.

Silva-Pinto, V, B. Arriaza y V. Standen 2010. Evaluación de la frecuencia de espina bífida oculta y su posible relación con el arsénico ambiental en una muestra prehispánica de la quebrada de Camarones, norte de Chile. Revista Médica de Chile 138:461-469.

Souffez, M.F. 1986. Los piojos en el mundo prehispánico, según algunos documentos de los siglos XV y XVI y unas representaciones en ceramios mochicas. Anthropologica 4:155-190.

Ubelaker, D. 1978. Human Skeletal Remains: Excavation, Analysis, Interpretation. Aldine Publishing, Chicago.

Ulloa, L., V. Standen y V. Gavilán 2000. Estudio de una prenda textil asociada al Inca en la costa norte de Chile (Camarones 9): las "mantas" que envuelven los cuerpos. Chungara Revista de Antropología Chilena 32 (2):259-261.

Weiss, R. 2009. Apes, lice and prehistory. Journal of Biology 8:20.

White, T. y P. Folkens 1991. Human Osteology. Academic Press, San Francisco. 